\title{
Important Announcement: Online Submission and Refereeing System Is Launched
}

(C) Springer Science + Business Media, LLC 2009

We are pleased to announce the launch of the Employee Responsibilities and Rights Journal web-based online manuscript submission and peer-review system. We invite all authors to submit their manuscripts to the journal's Editor-in-Chief, Margaret H. Vickers, via this online system, whose web address is:

\section{http://errj.edmgr.com}

The online system offers authors easy straightforward log-in and submission. Authors may submit their manuscripts in any of a wide range of file formats [such as Word, WordPerfect, RTF, TXT, and LaTeX for manuscripts; TIFF, GIF, JPEG, EPS, PPT, and Postscript for figures (artwork)]. The system offers a help feature, should an author encounter any difficulty when submitting. Authors may check the status of their manuscripts at any time throughout the refereeing process.

We are pleased to make available this state-of-the-art system, another service enhancement provided to authors. Most papers currently in the traditional system will continue their review process there. However, we urge all authors to submit their new manuscripts to the journal via this web-based online system. As usual, proposals for the Perspectives section should be sent via e-mail to the Perspectives Editor, Victor Devinatz. Complete author instructions may be found on the journal's homepage (http://www. springer.com/10672).

Authors, editors, and referees will save postage costs and all will enjoy speedier review times with the elimination of manuscript and disk mailing - not only at submission, but also throughout the refereeing/review process.

We have always invited the academic and professional community to submit their original papers to this excellent journal. Now we invite the community to submit their papers via the journal's online system, which provides users with convenience, speed, and cost savings throughout the submission and refereeing process.

The Editors and Publisher 